\title{
Expression of desmoglein 3 in non-small cell lung cancer distinguishes squamous cell lung carcinoma from adenocarcinoma.
}

Shahan Mamoor, MS1

1shahanmamoor@gmail.com

East Islip, NY USA

Non-small cell lung cancer, classified as adenocarcinomas or squamous cell lung carcinomas, is the major cause of cancer death in the United States and worldwide ${ }^{1}$. To understand the most significant transcriptional differences between adenocarcinomas and squamous cell lung carcinomas, we mined published microarray data from two separate studies ${ }^{2,3}$. We identified desmoglein 3 (DSG3) as a distinguishing transcriptional feature of squamous cell lung carcinomas, suggesting the biology of DSG3 may be relevant to the pathways critical for the development or maintenance of squamous cell lung carcinomas but not of adenocarcinomas. DSG3 expression was significantly correlated with prognosis of patients with NSCLC, with patients expressing low levels of DSG3 possessing significantly longer median overall survival. These analyses will also provide novel tools for diagnostic approaches and for guidance of treatment regimens for a cancer with dismal outlook.

Keywords: DSG3, desmoglein 3, non-small cell lung cancer, adenocarcinoma, squamous cell lung carcinoma, systems biology of NSCLC, targeted therapeutics in NSCLC, targeted diagnostics in NSCLC. 
Non-small cell lung cancer is the single most common cancer in the United States and the most common cause of cancer death in the United States ${ }^{1}$. The two major types of nonsmall cell lung cancer are adenocarcinoma and squamous cell lung carcinoma ${ }^{4}$.

Treatment approaches and prognosis can differ based on whether a patient is diagnosed with adenocarcinoma or squamous cell lung carcinoma ${ }^{4}$, and current approaches to distinguishing adenocarcinoma from squamous cell lung carcinoma rely on time-consuming procedures involving immunohistochemical staining with multiple markers including TTF1, p40, p63, CK5 and/or CK7 and subsequent readout by pathologists who will proceed to label each section, in a binary fashion, as positive or negative for each marker ${ }^{5}$. To understand the molecular nature of ACC and SCLC tumors in an unbiased fashion and the systems level, and to facilitate discovery of genes with utility in NSCLC diagnostics in a quantitative real-time workflow providing a quantitative and independent measure rather than qualitative measure based on pathologist interpretation, we performed comparative transcriptome analysis ${ }^{2,3}$ of the two major types of NSCLC tumors: adenocarcinomas and squamous cell lung carcinomas.

This blind, systems-level approach identified the gene encoding desmoglein 3, DSG3, as among the genes most differentially expressed when comparing adenocarcinomas and squamous cell lung carcinoma tumors, with DSG3 expression distinguishing squamous cell lung carcinomas from adenocarcinomas, suggesting DSG3 could be a useful tool in NSCLC diagnostics.

\section{Methods}

We utilized datasets GSE $74706^{2}$ and GSE $33532^{3}$ for this comparative transcriptome analysis of adenocarcinomas and squamous cell lung carcinomas. GSE74706 was generated using Agilent-026652 Whole Human Genome Microarray 4x44K v2 technology; for this analysis, we used $n=10$ adenocarcinomas and $n=4$ squamous cell lung carcinomas, and the analysis was performed using platform GPL13497. GSE33532 was generated using Affymetrix Human Genome U133 Plus 2.0 Array technology; for this analysis, we used $n=10$ adenocarcinoma tumors and $n=8$ squamous cell lung carcinomas, and the analysis was performed using platform GPL570.

The Benjamini and Hochberg method of $p$-value adjustment was used for ranking of differential expression but raw $p$-values were used to assess statistical significance of global differential expression. Log-transformation of data was auto-detected, and the NCBI generated category of platform annotation was used. A statistical test was performed to evaluate whether DSG3 expression was significantly between adenocarcinoma and squamous cell carcinoma using a two-tailed, unpaired t-test with Welch's correction. We used PRISM for all statistical analyses of differential gene expression in NSCLC tumors (Version 8.4.0)(455). For Kaplan-Meier survival analysis, we used the Kaplan-Meier plotter online tool ${ }^{7}$ for correlation of DSG3 mRNA expression levels with overall survival in $n=1925$ non-small cell lung cancer patients. 


\section{Results}

We mined published microarray data from two separate studies ${ }^{2,3}$ to describe in an unbiased fashion and at the systems level genes whose expression was most specific to either one of the two major types of non-small cell lung cancer: adenocarcinomas and squamous cell lung carcinomas.

\section{Desmoglein 3 expression distinguishes squamous cell lung carcinomas from adenocarcinomas.}

By comparing the global gene expression profiles of squamous cell lung carcinomas to adenocarcinomas ${ }^{2}$, we found that desmoglein 3 was the single most differentially expressed gene between the two major sub-types of NSCLC (Table 1). When sorting each of the genes expression by microarray based on significance of change in expression between adenocarcinomas and squamous cell lung carcinomas, desmoglein 3 ranked 1 out of 34183 total transcripts (Table 1). Desmoglein 3 differential expression between adenocarcinomas and squamous cell lung carcinomas was statistically significant (Table $1 ; p=6.6 \mathrm{E}-17$ ).

In a separate dataset ${ }^{3}$, desmoglein 3 was again the single most differentially expressed when comparing squamous cell lung carcinomas to adenocarcinomas in NSCLC (Table 2). When sorting each of the genes expression by microarray based on significance of change in expression between adenocarcinomas and squamous cell lung carcinomas, desmoglein 3 ranked 1 out of 25906 total transcripts (Table 2). Desmoglein 3 differential expression between adenocarcinomas and squamous cell lung carcinomas was again statistically significant (Table $2 ; p=6.18 \mathrm{E}-51)$.

\section{Desmoglein 3 is expressed at significantly higher levels in squamous cell lung} carcinomas than in adenocarcinomas.

We obtained exact mRNA values for desmoglein 3 to understand the magnitude and direction of difference in DSG3 mRNA expression between adenocarcinomas and squamous cell lung carcinomas in NSCLC. Desmoglein 3 was expressed at significantly higher levels in squamous cell lung carcinomas as compared to adenocarcinomas, and this difference was statistically significant (Figure 1 and Figure 2: $p<0.0001$ and $p<0.0001$, respectively). We calculated a mean fold change of $3.996 \pm 0.134$ in DSG3 expression when comparing squamous cell lung carcinomas to adenocarcinomas (Table 2).

\section{Expression of desmoglein 3 is associated with patient survival in NSCLC.}

We performed Kaplan Meier survival analysis to determine if desmoglein 3 expression was correlated with patient outcomes in NSCLC. We found significant correlation between expression of desmoglein 3 and overall survival in patients with NSCLC (Figure 3 and Table 3). While median overall survival was 90 months for NSCLC patients with low expression of DSG3, median overall survival was 53 months for NSCLC patients with high expression of DSG3. Correlation of desmoglein 3 tumor expression with median OS with statistically significant (Figure 3; log rank p-value: 2.4e-09; hazard ratio: 1.47 (1.29-1.67)). 
Thus, by mining published microarray data from independent datasets, we found that desmoglein 3 expression was among the genes whose expression most significantly distinguished squamous cell lung carcinomas from adenocarcinomas in NSCLC. Moreover, we found a statistically significant correlation between DSG3 tumor expression at the mRNA level and patient survival in NSCLC.

\section{Discussion}

To understand the most striking transcriptional features of each of the two major tumor types in NSCLC, we performed comparative transcriptome analysis of ACC and SCLC tumors using published microarray data ${ }^{2,3}$, discovering desmoglein 3 as the single most distinguishing transcriptional feature of squamous cell lung carcinomas.

Desmoglein 3 is a cell adhesion receptor and transmembrane glycoprotein of the cadherin family, functioning in desmosomes ${ }^{8}$. Desmoglein 3 is expressed in the basal and immediate suprabasal cell layers of the skin in keratinocytes ${ }^{9}$. Desmoglein 3 is also known as the pemphigus vulgaris antigen; patients with pemphigus vulgaris possess autoantibodies against desmoglein $3^{8}$. In addition to its role in cell adhesion in the deep squamous epithelium, desmoglein 3 also functions in anchoring telogen hair to a portion of the hair follicle known as the outer root sheath to maintain structural integrity ${ }^{10}$. Accodingly, mice deficient in DSG3 develop a phenotype similar to patients with pemphigus vulgaris, with separation of desmosomes visible by electron microscopy, and with both oral lesions and crusted skin possessing signs of suprabasilar acantholysis ${ }^{11}$.

While a previous study has described expression of desmoglein 3 in squamous cell lung carcinomas but not in adenocarcinomas, our analysis is significantly different than published findings ${ }^{12}$. First, our analysis utilized quantitative measures of desmoglein 3 at the RNA level, from two separate patient cohorts, while previously Fukuoka et al. measured desmoglein 3 at the protein level using immunohistochemical techniques with a distribution score of 1,2 , or 3 , and an intensity score of 1,2 , or 3 . Second, and more importantly, Fukuoka et al. described shorter survival for patients with "negative" histochemical staining of desmoglein 3 . On the other hand, after determining that desmoglein 3 was differentially expressed at the transcriptome level based on blind comparison to a reference transcriptome from the tissue of origin, the lung, we compared survival outcomes based on high and low expression, rather than positive and negative expression, and found that patients whose tumors expressed low levels of desmoglein 3, and at the RNA level, possessed significantly longer overall survival. Thus, while previous work has described specific expression of DSG3 in squamous cell lung carcinomas, our analysis describes differences in expression of DSG with more sensitivity and arrives at an opposite conclusion with respect to survival outcomes based on DSG3 tumor expression in patients with NSCLC.

We found that desmoglein 3 was the single most differentially expressed gene when comparing the tumors of patients with the two most common sub-types of NSCLC: adenocarcinomas and squamous cell lung carcinomas. We also found that desmoglein 3 expression significantly correlated with overall survival in patients with NSCLC. Desmoglein 3 has value as a diagnostic tool, as a prognostic indicator, and the biology of desmoglein 3 may be of value in understanding fundamental differences between the two major types of the most common type of cancer, and the most common cause of cancer death in the United States and worldwide. 


\section{References}

1. Siegel, R.L., Miller, K.D. and Jemal, A., 2019. Cancer statistics, 2019. CA: a cancer journal for clinicians, 69(1), pp.7-34.

2. Marwitz, S., Depner, S., Dvornikov, D., Merkle, R., Szczygieł, M., Müller-Decker, K., Lucarelli, P., Wäsch, M., Mairbäurl, H., Rabe, K.F. and Kugler, C., 2016. Downregulation of the TGF $\beta$ pseudoreceptor BAMBI in non-small cell lung cancer enhances TGF $\beta$ signaling and invasion. Cancer research, 76(13), pp.3785-3801.

3. Kabbout, M., Garcia, M.M., Fujimoto, J., Liu, D.D., Woods, D., Chow, C.W., Mendoza, G., Momin, A.A., James, B.P., Solis, L. and Behrens, C., 2013. Ets2 mediated tumor suppressive function and met oncogene inhibition in human non-small cell lung cancer. Clinical cancer research, 19(13), pp.3383-3395.

4. Cetin, K., Ettinger, D.S., Hei, Y.J. and D O'Malley, C., 2011. Survival by histologic subtype in stage IV nonsmall cell lung cancer based on data from the Surveillance, Epidemiology and End Results Program. Clinical epidemiology, 3, p.139.

5. Yuan, M., Huang, L.L., Chen, J.H., Wu, J. and Xu, Q., 2019. The emerging treatment landscape of targeted therapy in non-small-cell lung cancer. Signal Transduction and Targeted Therapy, 4(1), pp.1-14.

6. Osmani, L., Askin, F., Gabrielson, E. and Li, Q.K., 2018, October. Current WHO guidelines and the critical role of immunohistochemical markers in the subclassification of non-small cell lung carcinoma (NSCLC): Moving from targeted therapy to immunotherapy. In Seminars in cancer biology (Vol. 52, pp. 103-109). Academic Press.

7. Gyorffy, B., Surowiak, P., Budczies, J. and Lanczky, A., 2013. Online survival analysis software to assess the prognostic value of biomarkers using transcriptomic data in non-smallcell lung cancer. PloS one, 8(12), pp.e82241-e82241.

8. Amagai, M., Klaus-Kovtun, V. and Stanley, J.R., 1991. Autoantibodies against a novel epithelial cadherin in pemphigus vulgaris, a disease of cell adhesion. Cell, 67(5), pp.869-877.

9. Amagai, M., Koch, P.J., Nishikawa, T. and Stanley, J.R., 1996. Pemphigus vulgaris antigen (desmoglein 3 ) is localized in the lower epidermis, the site of blister formation in patients. Journal of Investigative Dermatology, 106(2), pp.351-355.

10.Koch, P.J., Mahoney, M.G., Cotsarelis, G., Rothenberger, K., Lavker, R.M. and Stanley, J.R., 1998. Desmoglein 3 anchors telogen hair in the follicle. Journal of Cell Science, 111(17), pp. 2529-2537.

11.Koch, P.J., Mahoney, M.G., Ishikawa, H., Pulkkinen, L., Uitto, J., Shultz, L., Murphy, G.F., Whitaker-Menezes, D. and Stanley, J.R., 1997. Targeted disruption of the pemphigus vulgaris antigen (desmoglein 3 ) gene in mice causes loss of keratinocyte cell adhesion with a phenotype similar to pemphigus vulgaris. The Journal of cell biology, 137(5), pp.1091-1102 
2

3

4

5

6

7

8

9

10

11

12

13

14

15

16

17

18

19

20

21

22

23

24

25

26

27

28

12.Fukuoka, J., Dracheva, T., Shih, J.H., Hewitt, S.M., Fujii, T., Kishor, A., Mann, F., Shilo, K., Franks, T.J., Travis, W.D. and Jen, J., 2007. Desmoglein 3 as a prognostic factor in lung cancer. Human pathology, 38(2), pp.276-283.

6 OF 12 

comparing whole transcriptomes of adenocarcinomas and squamous cell lung carcinomas, the two major types of non-small cell lung cancer.

Rank of differential expression, probe ID, $p$-value with respect to differential expression, $\mathrm{t}$, a moderated t-statistic, $\mathrm{B}$, the log-odds of differential expression between the two groups compared, and gene are listed in this chart.

\begin{tabular}{|c|c|c|c|c|c|}
\hline Rank & ID & p-value & $\mathbf{t}$ & B & Gene \\
\hline 1 & A_33_P3348747 & $6.6 \mathrm{E}-17$ & 28.0644355 & 23.26071 & DSG3 \\
\hline
\end{tabular}

Table 1: Desmoglein 3 is the single most differentially expressed gene when groups compared, and gene are listed in this chart. 

comparing whole transcriptomes of adenocarcinomas and squamous cell lung carcinomas, the two major types of non-small cell lung cancer.

Rank of differential expression, probe ID, $p$-value with respect to differential expression, $\mathrm{t}$, a moderated t-statistic, B, the log-odds of differential expression between the two groups compared, fold change in DSG3 expression when comparing squamous cell lung carcinomas to adenocarcinomas, gene, gene name and chromosomal location are listed in this chart.

8 OF 12 


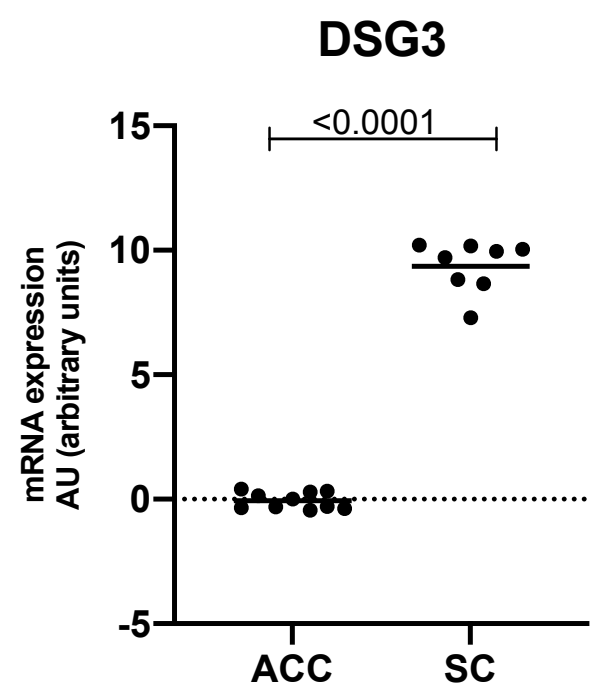

Figure 1: Expression of desmoglein 3 distinguishes squamous cell lung carcinoma from adenocarcinoma.

Messenger RNA (mRNA) levels of desmoglein 3 in the tumors of patients with adenocarcinoma ("ACC"; left) and in the tumors of patients with squamous cell lung carcinoma ("SC"; right) are graphically represented here with mean mRNA levels marked and the result of a statistical test evaluating significance of difference in mRNA expression between the tumors of patients with adenocarcinomas and squamous cell lung carcinomas, a $p$-value, listed above.

9 OF 12 


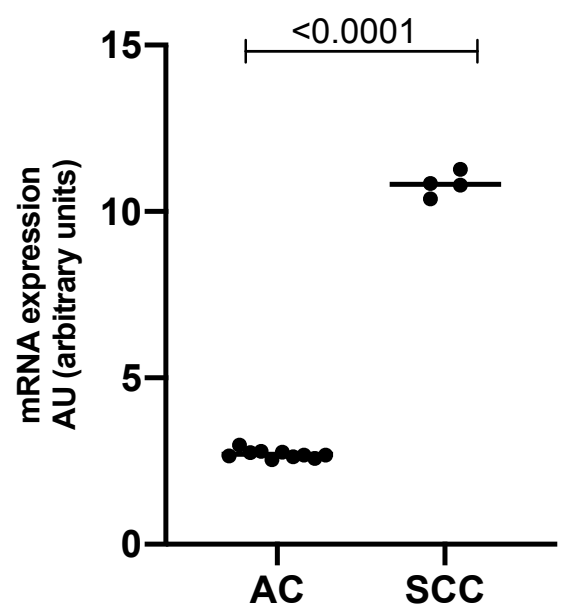

Figure 2: Expression of desmoglein 3 distinguishes squamous cell lung carcinoma from adenocarcinoma.

Messenger RNA (mRNA) levels of desmoglein 3 in the tumors of patients with adenocarcinoma ("ACC"; left) and in the tumors of patients with squamous cell lung carcinoma ("SC"; right) are graphically represented here with mean mRNA levels marked and the result of a statistical test evaluating significance of difference in mRNA expression between the tumors of patients with adenocarcinomas and squamous cell lung carcinomas, a $p$-value, listed above. 
Figure 3: Desmoglein 3 expression in the tumors of patients with NSCLC correlates with overall survival.

Depicted in this Kaplan-Meier plot is the probability of overall survival for $n=1925$ total patients stratified into two groups, based on low or high expression of DSG3 in patient tumors. The log rank $p$-value denoting statistical significance of difference in overall survival when comparing the two groups, as well as hazard ratio for this comparison is listed above. Listed below is the number of patients at risk (number of patients alive) per interval, after stratification based on DSG3 expression; in the first interval, number at risk is number of patients alive; in each subsequent interval, number at risk is the number at risk less those who have expired or are censored. 
Table 3: NSCLC patients with low tumor expression of DSG3 possess significantly greater overall survival than patients with high tumor expression of DSG3.

The median overall survival of $n=1925$ NSCLC patients based on stratification into low or high expression of DSG3 in tumors is listed in this chart. 